\title{
Real-world cost analysis of chemotherapy for colorectal cancer in Japan: detailed costs of various regimens during the entire course of chemotherapy
}

Shuichi Yajima ${ }^{1,2^{*}}$, Hisanori Shimizu ${ }^{3}$, Hiroyuki Sakamaki ${ }^{4}$, Shunya Ikeda ${ }^{5}$, Naoki Ikegami ${ }^{6}$ and Jun-lchiro Murayama ${ }^{7}$

\begin{abstract}
Background: Various chemotherapy regimens for advanced colorectal cancer have been introduced to clinical practice in Japan over the past decade. The cost profiles of these regimens, however, remain unclear in Japan. To explore the detailed costs of different regimens used to treat advanced colorectal cancer during the entire course of chemotherapy in patients treated in a practical setting, we conducted a so-called "real-world" cost analysis.

Method: A detailed cost analysis was performed retrospectively. Patients with advanced colorectal cancer who had received chemotherapy in a practical healthcare setting from July 2004 through October 2010 were extracted from the ordering system database of Showa University Hospital. Direct medical costs of chemotherapy regimens were calculated from the hospital billing data of the patients. The analysis was conducted from a payer's perspective.

Results: A total of 30 patients with advanced colorectal cancer were identified. Twenty patients received up to second-line treatment, and 8 received up to third-line treatment. The regimens identified from among all courses of treatment in all patients were 13 oxaliplatin-based regimens, 31 irinotecan-based regimens, and 11 regimens including molecular targeted agents. The average (95\% confidence interval [95 \% Cl]) monthly cost during the overall period from the beginning of treatment to the end of treatment was 308,363 $(258,792$ to 357,933) Japanese yen (JPY). According to the type of regimen, the average monthly cost was 418,463 (357,413 to 479,513) JPY for oxaliplatin-based regimens, 215,499 (188,359 to 242,639) JPY for irinotecan-based regimens, and 705,460 $(586,733$ to 824,187$)$ JPY for regimens including molecular targeted agents. Anticancer drug costs and hospital fees accounted for 50 to $77 \%$ and 11 to $25 \%$ of the overall costs of chemotherapy, respectively.

Conclusion: The costs of irinotecan-based regimens were lower than those of oxaliplatin-based regimens and regimens including molecular targeted agents in Japan. Using a lower cost regimen for first-line treatment can potentially reduce the overall cost of chemotherapy. The main cost drivers were the anticancer drug costs and hospitalization costs.
\end{abstract}

Keywords: Cost analysis, Colorectal cancer, Real-world setting, FOLFOX, FOLFIRI, XELOX, IRIS, Molecular targeted agents

\footnotetext{
* Correspondence: s-yajima@nifty.com

${ }^{1}$ Department of Health Policy and Management, School of Medicine, Keio

University, 35, Shinanomachi, Shinjuku-ku, Tokyo 160-8582, Japan

${ }^{2}$ Taiho Pharmaceutical Co., Ltd., 1-27, Kandanishiki-cho, Chiyoda-ku, Tokyo

101-8444, Japan

Full list of author information is available at the end of the article
} 


\section{Background}

Colorectal cancer is currently one of the most common cancers worldwide [1]. In Japan the incidence of colorectal cancer was 119,000 in 2010 [2], and there were 48,000 deaths from the disease in 2013 [3]. The disease is already advanced at the time of diagnosis in $56 \%$ of patients [2]. Surgery is the treatment of choice for early-stage colorectal cancer, while most patients with advanced colorectal cancer receive chemotherapy.

A combination of 5-fluorouracil (5-FU) and leucovorin (LV) was the standard regimen for advanced colorectal cancer during the 1980s and 1990s. After the year 2000, FOLFOX (oxaliplatin plus 5-FU and LV) and FOLFIRI (irinotecan plus 5-FU and LV) were developed and modified over the course of several years. These regimens extended progression-free survival to more than 8 months [4]. Furthermore, regimens including molecular targeted agents such as bevacizumab (Bev) or cetuximab (Cet) have prolonged progression-free survival to 9 to 11 months [5]. Currently, both FOLFOX and FOLFIRI with or without molecular targeted agents are commonly used to manage advanced colorectal cancer throughout the world [6-8]. Meanwhile, regimens including easy-to-administer oral agents were demanded, because both FOLFOX and FOLFIRI require continuous infusion of 5-FU for 2 days, which was not convenient in practice. A regimen combining the oral agent capecitabine with oxaliplatin (XELOX) was developed for the treatment of colorectal cancer. Two phase III trials showed that progression-free survival of patients who received XELOX is non-inferior to that of patients who received FOLFOX $[9,10]$, and XELOX is now used worldwide. In Japan, a regimen combining oral S-1 (a combination of tegafur, gimeracil, and oteracil), which was already widely used to treat gastric cancer, with irinotecan (IRIS) was developed for the management of advanced colorectal cancer [11]. The FIRIS study demonstrated that the progression-free survival of patients in the IRIS group was non-inferior to that in the FOLFIRI group [12], and IRIS is now one treatment option for this disease.

Despite the usefulness of various regimens for the treatment of advanced colorectal cancer, the development of new combination regimens has also attracted the attention of payers as well as physicians owing to the high costs of chemotherapy for colorectal cancer. The national medical expenditure in Japan reached 39.2 trillion JPY in 2011 [13]. Above all, the growing costs of cancer treatment are a burden to both the national medical expenditure and the out-of-pocket costs paid by patients. A number of researchers have reported the results of economic evaluations of colorectal cancer treatment in Japan [14-16]. All of these evaluations have provided useful information to decision makers and practitioners. However, the economic information in all except one [16] of these reports is insufficient for practical clinical use owing to the limited setting, such as clinical trials and controlled studies. In other words, cost-related data on the costs of chemotherapy in a practical clinical setting are thought to differ from data derived from planned clinical studies. Furthermore, the comprehensive economic profile of chemotherapy from first-line to the end of treatment for colorectal cancer is unclear in clinical trials. One task force of the International Society for Pharmacoeconomics and Outcomes Research (ISPOR) has reported the advantages of realworld data [17], and another task force of the ISPOR has provided a questionnaire to assess the relevance and credibility of observational studies designed to fill the gap between clinical trials and actual clinical practice [18].

We have recently reported the results of a costminimization analysis comparing $\mathrm{S}$-1-based regimens with 5FU-based regimens for advanced colorectal cancer in Japan [19]. In the present study, we focused on the comprehensive economic profile of chemotherapy and performed a detailed cost analysis of various regimens used to manage this disease from first-line treatment to the end of chemotherapy in a real-world setting.

\section{Methods \\ Analytical methods}

We undertook a real-world cost analysis to compare the detailed costs of different chemotherapy regimens used for advanced colorectal cancer from first-line treatment to the end of chemotherapy in a practical setting. The analysis was retrospectively performed from a payer's perspective. The costs of healthcare-related services were calculated from the payer side using billing data obtained from Showa University Hospital. Showa University Hospital is a general hospital located in Tokyo that has approximately 800 beds as well as outpatient clinics. The fees are the same for all payers and virtually all providers in Japan and are set by the government in the national fee schedule [20]. The study was approved by the Medical Ethics Committee of Showa University Hospital (approved number: 981).

We retrospectively collected clinical and claim data on patients with advanced colorectal cancer in this observational study. Because most of the patients died before this study began, it was difficult to obtain written consent from the patients. We analyzed the data after removing all personal information to protect privacy. This approach was in accordance with the Japanese Ethical Guidelines for Epidemiological Studies [21]; moreover, before the study began the Internal Review Board determined that consent is not required. 


\section{Patients and chemotherapy regimens}

The study group comprised patients who were given a diagnosis of advanced colorectal cancer and received chemotherapy until the end of the final line of chemotherapy in the Department of Surgery, Showa University Hospital during the period from July 2004 through October 2010. We extracted data on all lines of chemotherapy given to all patients and classified the regimens into the following three groups: oxaliplatin-based regimens, irinotecan-based regimens, and regimens including molecular targeted agents.

\section{Resource utilization data and costs}

Resource utilization data on each chemotherapy regimen was extracted from Showa University Hospital billing data. The following costs were calculated on a monthly basis and were summed up on the basis of the Japanese National Health Insurance fee-for-service system (reimbursement price) in 2010: costs related to outpatient visits (physician consultations and outpatient visits); costs for hospitalization (basic bed charges, medical examinations, nursing care, and basic treatments during hospitalization); costs for operations and procedures (including transfusions); costs for laboratory tests and diagnostic imaging tests (radiography, computed tomography, magnetic resonance imaging, ultrasonography); costs for drugs and administration (anticancer drugs, antiemetics, and other drugs, including preparation and administration costs). Costs unrelated to cancer, such as costs required for the management of hypertension and hyperlipidemia, and operation fees, such as those required for the creation of an artificial anus, were not considered. Extra bed fees and meal fees were collected from billing data as a reference.

In this study, we collected data on regimens regardless of the treatment line, and then found that the duration of each regimen was influenced by the treatment line. The duration of the first line of treatment was longer than those of second and third lines. Therefore, the costs of each chemotherapy regimen were compared on a monthly basis to avoid the effects of the different durations of treatment.

\section{Time horizon and discounts}

The monthly average costs of all chemotherapy regimens from first-line to the end of the final line of treatment were analyzed over the course of time. Treatments costs during the period of terminal care were not considered because it was difficult to collect relevant data during that period, and the costs varied depending on the status of individual patients during terminal care, regardless of the regimens used previously. Trends in the breakdown of costs were also analyzed in detail. Costs for more than 1 year were discounted by $3 \%$.

\section{Sensitivity analysis}

The uncertainty of the results was explored by sensitivity analyses of uncertain factors. Several qualitative, one-way sensitivity analyses were conducted to explore the impact of alternative parametric assumptions on the results.

\section{Results}

Characteristics of patients and chemotherapy regimens

The characteristics of the 30 patients identified from the patient database of Showa University Hospital are shown in Table 1 . The average age was 62.8 years. The major sites of colorectal cancer were the cecum in 4 patients, the ascending colon in 3 , the transverse colon in 2 , the descending colon in 1 , the sigmoid colon in 7 , and the rectum in 13 . The rate of hospitalization was $23 \%$. The average duration of the entire course of chemotherapy was 13.5 months. Among the 30 patients, 20 received up to second-line chemotherapy, and 8 received up to third-line chemotherapy. The following different regimens were identified from among all courses of treatment in all patients: 13 oxaliplatin-based regimens (12 FOLFOX regimens and 1 XELOX regimen); 31 irinotecan-based regimens (27 IRIS regimens and 4 FOLFIRI regimens); 11 regimens including molecular targeted agents (6 FOLFOX + Bev regimens, 4 FOLFIRI + Bev or Cet regimens and 1 XELOX + Bev regimen); and 3 other regimens (2 Bev regimens and 1 Cet regimen). The schedules of each regimen are presented in Additional file 1.

The numbers of regimens and the durations of each treatment line are shown in Table 2. Two oxaliplatin-based regimens (2 FOLFOX regimens), 27 irinotecan-based regimens (27 IRIS regimens), and 1 regimen including molecular targeted agents (1 FOLFIRI + Bev regimen) were given as first-line treatment. Eleven oxaliplatin-based regimens (10 FOLFOX regimens, 1 XELOX regimen), 8 regimens including molecular targeted agents (6 FOLFOX + Bev regimens, 1 FOLFIRI + Cet regimen, 1 XELOX + Bev regimen) and 1 Bev regimen were given as second-line treatment. Four irinotecan-based regimens (4 FOLFIRI regimens), 2 regimens including molecular targeted agents (1 FOLFIRI + Bev regimen, 1 FOLFIRI + Cet regimen), 1 Bev regimen and 1 Cet regimen were given as third-line treatment. The average duration of each line was 9.0 months for first-line, 3.9 months for second-line, and 3.8 months for third-line. The average duration of chemotherapy according to the type of regimen was 3.9 months for oxaliplatin-based regimens, 8.8 months for irinotecan-based regimens, and 4.3 months for regimens including molecular targeted agents. 
Table 1 Patient characteristics

\begin{tabular}{|c|c|}
\hline Number of patients & 30 \\
\hline \multicolumn{2}{|l|}{ Sex } \\
\hline Male & 21 \\
\hline Female & 9 \\
\hline \multicolumn{2}{|l|}{ Age } \\
\hline-59 & 11 \\
\hline $60-69$ & 9 \\
\hline $70-$ & 10 \\
\hline Average & 62.8 \\
\hline \multicolumn{2}{|l|}{ Site of colorectal cancer } \\
\hline Cecum & 4 \\
\hline Ascending colon & 3 \\
\hline Transverse colon & 2 \\
\hline Descending colon & 1 \\
\hline Sigmoid colon & 7 \\
\hline Rectum & 13 \\
\hline Rate of hospitalization & $23 \%$ \\
\hline Overall treatment period (average) & 13.5 months \\
\hline \multicolumn{2}{|l|}{ Treatment lines } \\
\hline First-line & 30 \\
\hline Second-line & 20 \\
\hline Third-line & 8 \\
\hline \multicolumn{2}{|l|}{ Regimens } \\
\hline Oxaliplatin-based regimens & 13 \\
\hline FOLFOX & 12 \\
\hline XELOX & 1 \\
\hline Irinotecan-based regimens & 31 \\
\hline IRIS & 27 \\
\hline FOLFIRI & 4 \\
\hline Regimens including molecular targeted agents & 11 \\
\hline FOLFOX + Bev & 6 \\
\hline FOLFIRI + Bev or Cet & 4 \\
\hline XELOX + Bev & 1 \\
\hline Others & 3 \\
\hline
\end{tabular}

Overall treatment period $=$ from the beginning of treatment to the end of the final line of chemotherapy (All of the patients had died or were in terminal care phase as of the study endpoint)

The numbers of each regimen were counted from among all courses of treatment in all patients

FOLFOX oxaliplatin plus 5-fluorouracil and leucovorin; FOLFIRI irinotecan plus 5-fluorouracil and leucovorin; XELOX oxaliplatin plus capecitabine; IRIS irinotecan plus S-1 (tegafur, gimeracil and oteracil combination); Bev bevacizumab; Cet cetuximab; Others 2 Bev and 1 Cet

\section{Costs}

Total costs for the entire course of chemotherapy and costs according to regimen group

The monthly total costs are shown in Table 3 and Fig. 1, and the time courses of the total costs of each regimen group are shown in Fig. 2.
The average (95\% CI, minimum, 75th percentile, median, 75th percentile, maximum) monthly cost during the overall period from the beginning of treatment to the end of treatment was 308,363 (258,792 to 357,933 , 152,345, 208,653, 270,328, 340,179, 637,299) JPY. The monthly costs varied over time. The first cost peak occurred in the first month, and the cost gradually increased from the fifth to the twelfth month. During this period, some patients started to receive expensive secondline treatments. The second cost peak occurred in the twelfth month and overlapped with the peak in the first month of third-line treatment. The average (95\% CI, minimum, 75th percentile, median, 75 th percentile, maximum) monthly costs were 256,149 (207,992 to 304,307 , $149,816,186,293,208,555,261,922,637,299)$ JPY for firstline treatment, 518,897 (431,290 to $606,504,280,977$, 348,454, 431,758, 650,541, 925,339) JPY for second-line, and $416,251 \quad(188,316$ to $644,185,141,582,162,033$, 297,117, 600,389, 1,056,666) JPY for third-line.

The average (95\% CI, minimum, 75 th percentile, median, 75th percentile, maximum) monthly costs of oxaliplatin-based regimens, irinotecan-based regimens, and regimens including molecular targeted agents were respectively 418,463 (357,413 to $479,513,280,977,339,667$, $399,767,444,406,635,115)$ JPY, 215,499 (188,359 to 242,639, 141,867, 171,990, 207,516, 223,415, 567,549) JPY, and $705,460(586,733$ to $824,187,411,384,597,586,710,434$, $822,972,1,056,666)$ JPY. The costs of irinotecan-based regimens were lower than those of oxaliplatin-based regimens and regimens including molecular targeted agents.

\section{Cost-component analysis}

The monthly component costs of each regimen group up to the twelfth month of treatment are shown in Table 4.

The cost drivers were anticancer drugs and hospitalization. The costs of anticancer drugs were 228,539 JPY (proportion of total: $58 \%$ ) for oxaliplatin-based regimens, 96,853 JPY (50 \%) for irinotecan-based regimens, and 535,214 JPY (77 \%) for regimens including molecular targeted agents. The costs were lower for irinotecan-based regimens than for oxaliplatin-based regimens and regimens including molecular targeted agents. The hospital fees were 89,935 JPY (21 \%) for oxaliplatin-based regimens, 53,584 JPY (25 \%) for irinotecan-based regimens, and 77,752 JPY (11 \%) for regimens including molecular targeted agents. Next to the costs of anticancer drugs, hospital fees were second highest costs for all regimen groups.

The costs of other components were as follows. The administration costs for drug administration were 19,882 JPY for oxaliplatin-based regimens and 16,133 JPY for regimens including molecular targeted agents, which were higher than the 12,543 JPY for irinotecan-based regimens. 
Table 2 Numbers of regimens used in each treatment line and durations of each treatment line

\begin{tabular}{|c|c|c|c|c|}
\hline & First-line & Second-line & Third-line & Average durations of treatment (month) \\
\hline Total & 30 & 20 & 8 & 6.5 \\
\hline Oxaliplatin-based regimens & 2 & 11 & & 3.9 \\
\hline FOLFOX & 2 & 10 & & 3.8 \\
\hline XELOX & & 1 & & 5.0 \\
\hline Irinotecan-based regimens & 27 & & 4 & 8.8 \\
\hline IRIS & 27 & & & 9.3 \\
\hline FOLFIRI & & & 4 & 5.8 \\
\hline Regimens including molecular targeted agents & 1 & 8 & 2 & 4.3 \\
\hline FOLFOX + Bev & & 6 & & 5.0 \\
\hline FOLFIRI + Bev or Cet & 1 & 1 & 2 & 4.0 \\
\hline XELOX + Bev & & 1 & & 1.0 \\
\hline Others & & 1 & 2 & 1.3 \\
\hline Average durations of treatment (month) & 9.0 & 3.9 & 3.8 & \\
\hline
\end{tabular}

The diagnostic imaging cost for irinotecan-based regimens was 24,039 JPY, which was higher than the costs in the other regimen groups. The operation fees were 37,353 JPY for oxaliplatin-based regimens and 38,222 JPY for regimens including molecular targeted agents, which were higher than the operation fees for irinotecan-based regimens.

\section{Sensitivity analysis}

The results of sensitivity analysis are shown in Table 5 . As mentioned above, the treatment line differed among the regimens in this study. There was some bias, which most likely depended on the treatment line. Regimens used as first-line during the first month of treatment tended to be administered in the hospital, and the hospital fee accounted for a large proportion of total costs and might have influenced the results. We therefore initially performed a simple sensitivity analysis to examine the hospital fee. Two scenarios were considered: 3 days' hospitalization and an outpatient setting. As a result, the orders of the total costs among the regimen groups given on an inpatient basis and an outpatient basis were basically same as the order of the total costs in the regimen groups for the base-case. As for oxaliplatin-based regimens and regimens including molecular targeted agents, the base-case costs were slightly higher than those of a 3-day hospitalization setting because the period of hospitalization was longer than 3 days for the base-case. The costs for diagnostic imaging tests were higher for irinotecan-based regimens than for the

Table 3 Average monthly total costs (JPY)

\begin{tabular}{|c|c|c|c|c|c|c|c|}
\hline & Total costs (mean) & $95 \% \mathrm{Cl}$ & Minimum & 25th percentile & Median & 75th percentile & Maximum \\
\hline $\begin{array}{l}\text { Overall treatment period } \\
(n=30)\end{array}$ & 308,363 & $(258,792-357,933)$ & 152,345 & 208,653 & 270,328 & 340,179 & 637,299 \\
\hline $\begin{array}{l}\text { First-line treatment } \\
(n=30)\end{array}$ & 256,149 & $(207,992-304,307)$ & 149,816 & 186,293 & 208,555 & 261,922 & 637,299 \\
\hline $\begin{array}{l}\text { Second-line treatment } \\
(n=20)\end{array}$ & 518,897 & $(431,290-606,504)$ & 280,977 & 348,454 & 431,758 & 650,541 & 925,339 \\
\hline $\begin{array}{l}\text { Third-line treatment } \\
(n=8)\end{array}$ & 416,251 & $(188,316-644,185)$ & 141,582 & 162,033 & 297,117 & 600,389 & $1,056,666$ \\
\hline $\begin{array}{l}\text { Oxaliplatin-based regimens } \\
(n=13)\end{array}$ & 418,463 & $(357,413-479,513)$ & 280,977 & 339,667 & 399,767 & 444,406 & 635,115 \\
\hline $\begin{array}{l}\text { Irinotecan-based regimens } \\
(n=31)\end{array}$ & 215,499 & $(188,359-242,639)$ & 141,867 & 171,990 & 207,516 & 223,415 & 567,549 \\
\hline $\begin{array}{l}\text { Regimens including molecular targeted } \\
\text { agents }(n=11)\end{array}$ & 705,460 & $(586,733-824,187)$ & 411,384 & 597,586 & 710,434 & 822,972 & $1,056,666$ \\
\hline
\end{tabular}

Costs during the overall treatment period were calculated from the beginning of treatment to the end of treatment and were discounted by $3 \%$ after the twelfth month

The costs of first-line, second-line, and third-line treatments were calculated until the end of each treatment and were discounted by $3 \%$ after the twelfth month The costs of each regimen group were calculated until the twelfth month and were not discounted 


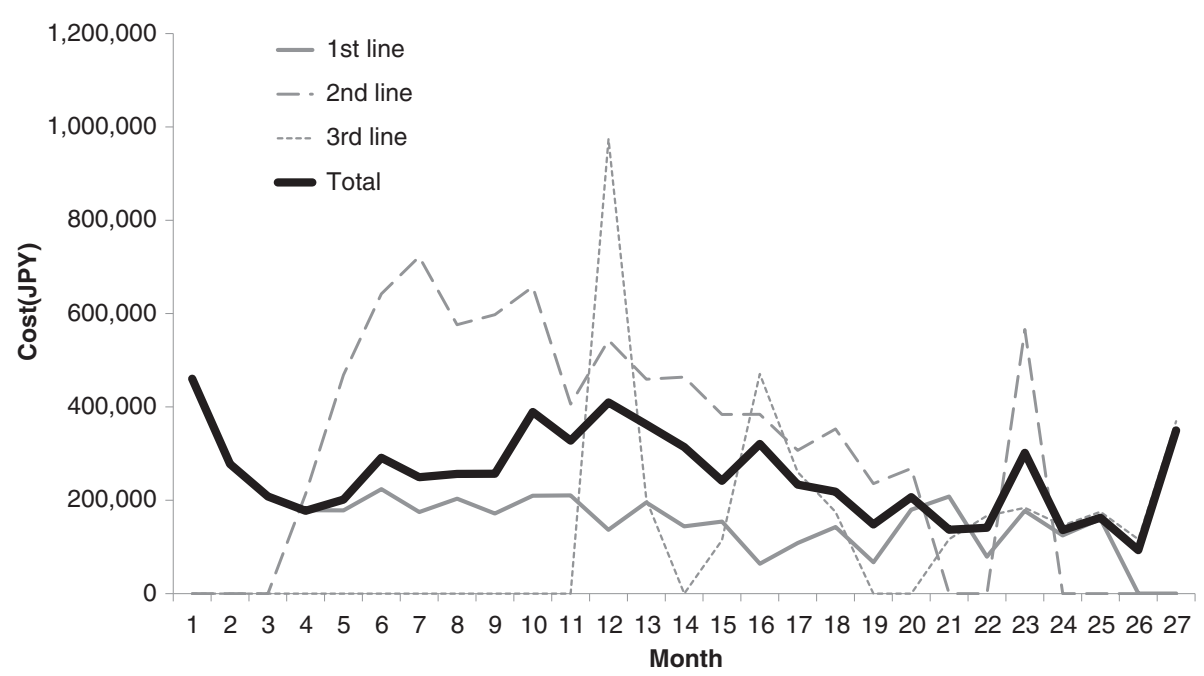

Fig. 1 Monthly total costs from the beginning of treatment to the end of treatment. The costs of each line and costs from the beginning of treatment to the end of treatment are shown. The earliest starting point of second-line treatment was the fourth month and that of third-line treatment was the twelfth month

other regimen groups because IRIS, which was the most commonly used irinotecan-based regimen, was mainly used for first-line treatment, during which diagnostic imaging tests were frequently performed to assess disease progression. Therefore, we performed a simple sensitivity analysis of the costs for diagnostic imaging tests. The results demonstrated that the cost of diagnostic imaging tests did not have an impact on the total costs. In addition, because the use of generic anticancer drugs may have influenced the results, we performed a simple sensitivity analysis comparing the use of generic leucovorin with that of brand leucovorin. The results did not change.

\section{Discussion}

Current clinical guidelines for colorectal cancer [6-8], recommend FOLFOX or FOLFIRI with or without molecular targeted agent such as Bev or Cet as first-line treatment. In the present study, however, the main regimen used for first-line treatment was the oral S-1-based IRIS regimen. IRIS was approved for reimbursement for colorectal

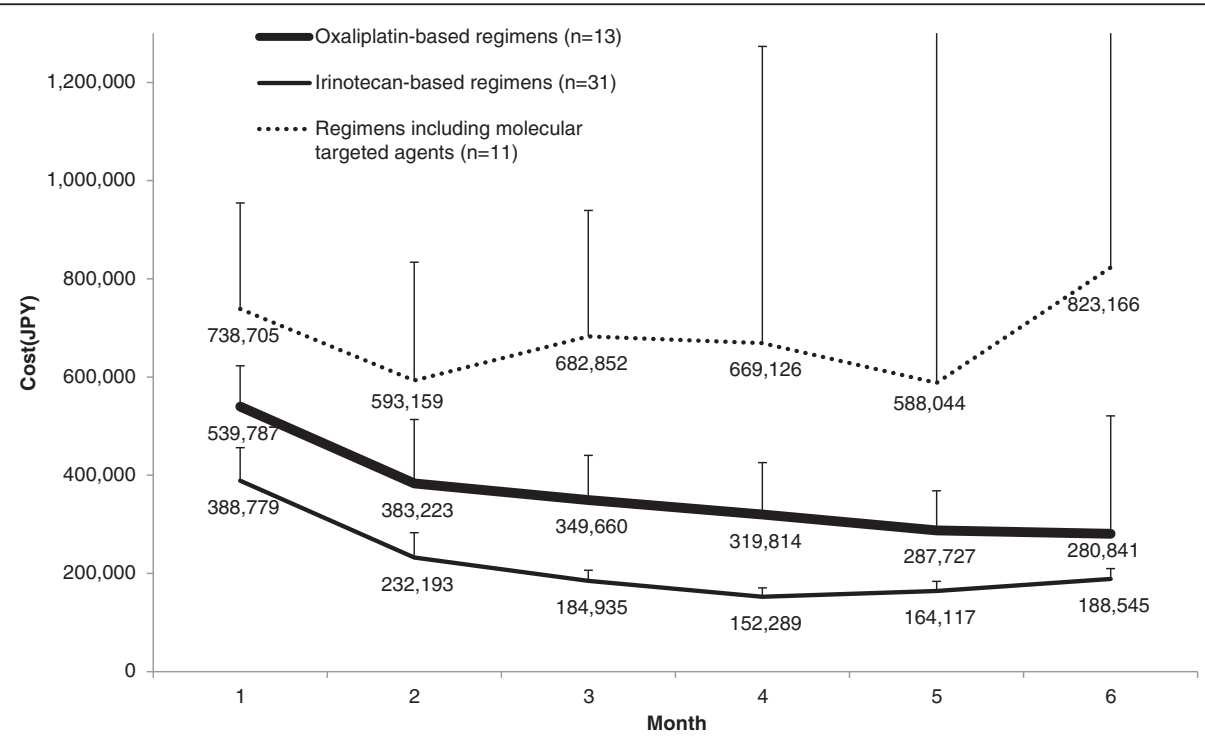

Fig. 2 Total costs of each regimen up to the sixth month (mean $+95 \% \mathrm{Cl})$ 
Table 4 Average monthly component costs of each regimen group up to the twelfth month (JPY)

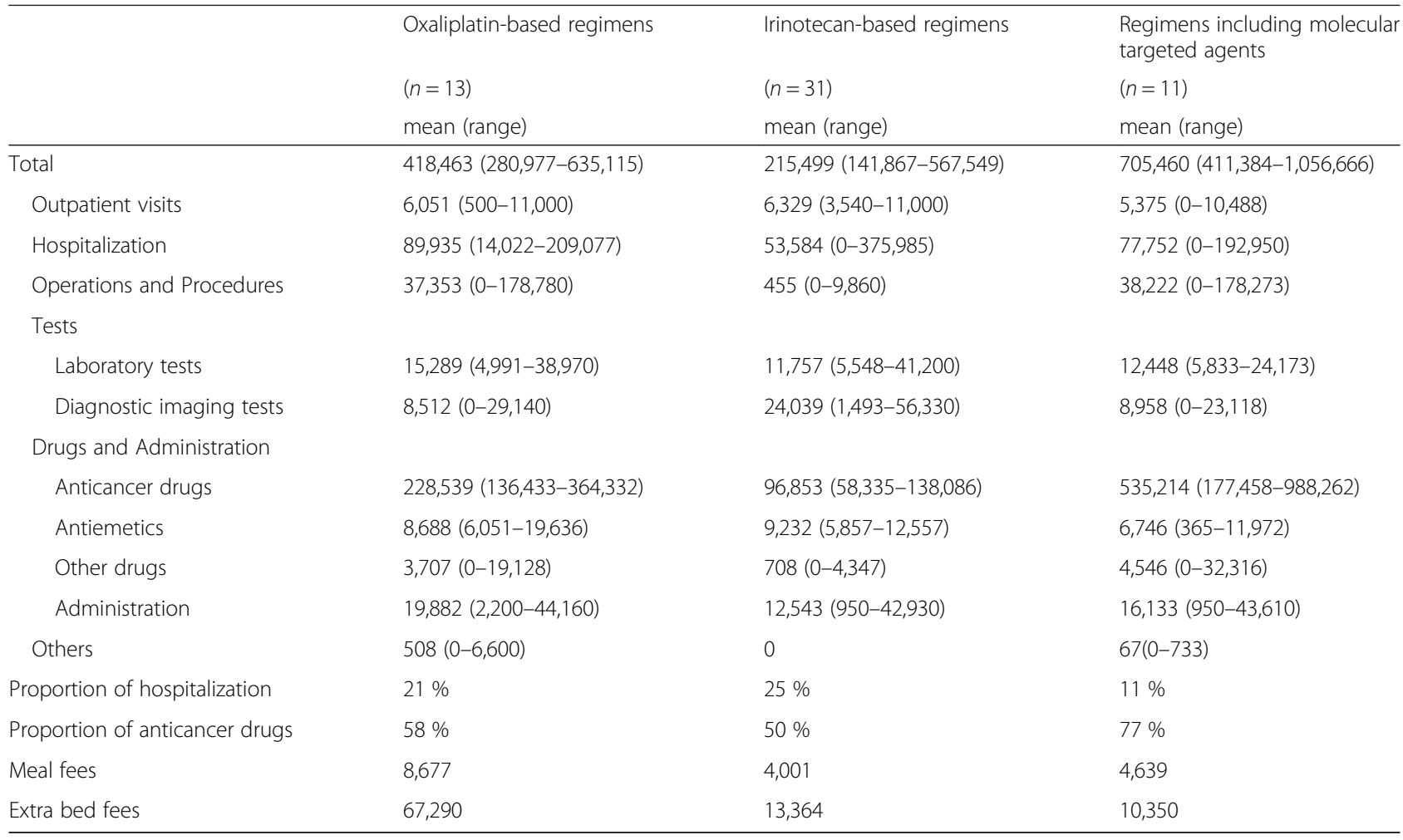

Outpatient visits = physician consultations and outpatient visits; Hospitalization = basic bed charges, medical examinations, nursing care, and basic treatments during hospitalization; Operations = including transfusion; Diagnostic imaging tests = X-ray, CT, MRI, ultrasound; Administration = preparation and management; Range $=$ minimum to maximum

cancer in 2003 under Japanese National Health Insurance before the present study began, and the FIRIS study comparing IRIS with FOLFIRI started in 2006 [12]. Therefore, physicians used the more convenient oral IRIS regimen as first-line chemotherapy during this study, because they expected IRIS to be as effective as FOLFIRI, one of the standard regimens used to treat advanced colorectal cancer. On the other hand, molecular targeted agents and XELOX were virtually not used as first-line treatment during this study, because these treatments were approved for reimbursement under Japanese National Health Insurance in 2007 and 2009, respectively, which was during the latter part of this study.

In the present study, the average duration of the entire course of chemotherapy was longer than 1 year, and the average monthly total cost during the entire period was 308,363 JPY, which nearly equals the average wage in Japan [22]. The burden to health insurance and individuals was thus substantial. The duration of first-line treatment was 9.0 months, which was longer than the duration of second and subsequent lines of chemotherapy. Therefore, the cost of the entire course of chemotherapy

Table 5 Sensitivity analysis (average monthly total costs: JPY)

\begin{tabular}{llll}
\hline & Oxaliplatin-based regimens & Irinotecan-based regimens & Regimens including molecular targeted agents \\
$(n=13)$ & $(n=31)$ & $(n=11)$ \\
\hline Base-case & 418,463 & 215,499 & 705,460 \\
Hospitalization & 390,487 & 223,597 & 690,343 \\
Outpatient & 327,177 & 160,287 & 627,033 \\
Imaging Max & 433,991 & 215,499 & 720,541 \\
Imaging Min & 418,463 & 199,972 & 705,013 \\
Brand drug & 418,463 & 215,888 & 707,715 \\
Generic drug & 404,097 & 214,046 & 694,883
\end{tabular}

Hospitalization = in the scenario of 3 days' hospitalization; Outpatient = in the scenario of outpatient visit; Imaging Max = in the scenario of maximum utilization of diagnostic imaging tests; Imaging Min = in the scenario of minimum utilization of diagnostic imaging tests; Brand drug = in the scenario of using brand leucovorin; Generic drug $=$ in the scenario of using generic leucovorin 
seemed to depend primarily on the regimen used for firstline treatment. In this study, inexpensive IRIS was used for first-line treatment, which contributed to a lower overall cost for chemotherapy.

The costs of oxaliplatin-based regimens such as FOLFOX were higher than those of irinotecan-based regimens such as IRIS and FOLFIRI. Regimens including molecular targeted agents such as Bev and Cet were the most expensive. Many economic evaluations of colorectal cancer chemotherapy have been performed in the world (Table 6). Miyazaki et al., Shiroiwa et al., and Ajima et al. have reported on the costs of chemotherapy for advanced colorectal cancer in Japan [14-16]. All of these studies showed that the cost of FOLFOX is higher than that of FOLFIRI, consistent with the results of our study. In the United States, several economic evaluations of the FOLFOX and FOLFIRI regimens have also reported that FOLFOX is more expensive than FOLFIRI [23, 24]. In the United Kingdom, however, the costs of FOLFOX and FOLFIRI are about the same [25, 26]. These differences seem to be attributed to the different drug prices in each country. As for molecular targeted agents, several economic evaluations of $\mathrm{Bev}$ have been reported by Shiroiwa et al., Tappenden et al., and Lee et al. [15, 27, 28].
These studies showed that the cost-effectiveness of Bev is low. In the United Kingdom, the National Institute for Health and Care Excellence (NICE) did not recommend molecular targeted agents as first-line treatment for advanced colorectal cancer [29].

Hospital fees were thought to be one of the cost drivers. However, the results of sensitivity analysis showed that the hospital fees did not influence the total costs. The basecase costs of oxaliplatin-based regimens and regimens including molecular targeted agents were higher than the cost of the scenario assuming 3 days of hospitalization. The management of adverse effects was apparently more difficult for oxaliplatin-based regimens and regimens including molecular targeted agents than for other regimens and thus required a longer hospitalization period.

The cost characteristics of other components were as follows. The costs associated with drug administration were higher for oxaliplatin-based regimens (FOLFOX, etc.) than for irinotecan-based regimens (IRIS, etc.) because IRIS regimens did not require continuous infusion of 5-FU. The diagnostic imaging costs for irinotecan-based regimens, used mainly as first-line treatment, were higher than those for other regimens because diagnostic imaging was frequently performed

Table 6 Costs of chemotherapy for advanced colorectal cancer in the world (USD, monthly)

\begin{tabular}{|c|c|c|c|}
\hline & Oxaliplatin-based regimens & Irinotecan-based regimens & $\begin{array}{l}\text { Regimens including molecular } \\
\text { targeted agents }\end{array}$ \\
\hline \multirow[t]{2}{*}{ Japan (Present study) } & FOLFOX: 4,141 & IRIS: 2,217 & FOLFOX + Bev: 7,353 \\
\hline & & FOLFIRI ( $2^{\text {nd }}$ line): 1,574 & FOLFIRI + Bev or Cet: 6,777 \\
\hline Japan (Miyazaki4) & FOLFOX: 4,151 & FOLFIRI: 2,398 & \\
\hline \multirow[t]{3}{*}{ Japan (Shiroiwa ${ }^{15}$ ) } & FOLFOX: 4,554 & FOLFIRI ( $2^{\text {nd }}$ line): 2,376 & FOLFOX + Bev: 7,722 \\
\hline & XELOX: 3,564 & & IFL + Bev: 4,950 \\
\hline & & & XELOX + Bev: 6,831 \\
\hline Japan $\left(\right.$ Ajima $\left.{ }^{16}\right)$ & FOLFOX: 5,352 & FOLFIRI: 3,743 & \\
\hline US (Mullins ${ }^{23}$ ) & FOLFOX: 60,179 (per patient) & FOLFIRI: 44,087 (per patient) & \\
\hline US (Tumeh ${ }^{24}$ ) & FOLFOX: 29,865 (per patient) & FOLFIRI: 24,551 (per patient) & \\
\hline \multirow[t]{2}{*}{ US $\left(\mathrm{Chu}^{30}\right)$} & FOLFOX: 14,300 & & \\
\hline & XELOX: 11,473 & & \\
\hline UK (NICE:HTA2001 ${ }^{25}$ ) & FOLFOX: 5,718 & FOLFIRI: 5,975 & \\
\hline UK (NICE:HTA200826) & FOLFOX: 22,734 (per patient) & FOLFIRI: 23,017 (per patient) & \\
\hline UK (Tappenden ${ }^{27}$ ) & & IFL: 40,998 (per patient) & IFL + Bev: 74,379 (per patient) \\
\hline \multirow[t]{2}{*}{ France (Perrocheau ${ }^{32}$ ) } & FOLFOX: 23,597 (per patient) & & \\
\hline & XELOX: 17,695 (per patient) & & \\
\hline Koria $\left(\right.$ Lee $\left.^{28}\right)$ & & FOLFIRI: 1,597 & Bev: 5,150 \\
\hline \multirow[t]{3}{*}{ UK $\left(\right.$ Ward $\left.^{31}\right)$} & 5-FU/LV (Mayo): 2,065 & & \\
\hline & Capecitabine: 1,225 & & \\
\hline & UFT/LV: 1,944 & & \\
\hline
\end{tabular}

IFL irinotecan plus fluorouracil and leucovorin; 5-FU 5-fluorouracil; LV leucovorin; UFT tegafur and uracil combination Rate: 1 USD (US Dollar) = 101 JPY, 0.58 GBP (British Pound), 0.73 EUR (Euro) (2014/7/9) 
during first-line treatment. Operation fees for placement of a central venous access port for continuous infusion of 5-FU were high for oxaliplatin-based regimens, because most FOLFOX regimens were used before FOLFIRI in this study. The costs of antiemetics did not differ among the regimens.

As reference information, we also collected data on the extra bed fee paid by patients, which was not included in total costs in this study. The price depended on the type of room, irrespective of the type of regimen. Such out-of-pocket costs paid by the patient, however, were a great burden in a practical setting. We need to consider such out-of-pocket costs from a societal perspective in future studies.

Our analysis had several important limitations. First, a small sample of claim data was collected from a single institution in this study. It seems to be difficult to generalize the results of this study. However, standard dosages and schedules of the regimens in Showa University Hospital were used. In addition, by grouping the regimens to compensate for the small numbers of patients who received each regimen, we could obtain convincing results to some extent. Second, the treatment line differed among the regimens. However, we did not attempt to balance the background characteristics of the patients according to each regimen, because treatment dosages and schedules did not differ between the lines. With regard to this point, the durations and total costs of each regimen group varied according to the treatment line. Therefore, we compared the regimen groups on a monthly basis to eliminate potential effects related to differences in the duration of each treatment. Ideally, however, we have to directly compare total costs in a clinical trial. Third, we calculated only direct costs because our analysis was performed from a payer's perspective.

As mentioned above, the most widely used regimen in this study was oral S-1-based IRIS. Several economic evaluations of regimens including oral preparations such as capecitabine and tegafur-uracil have shown that such regimens are more cost effective than regimens comprising only injectable preparations [15, 30-32]. In our former study analyzing treatment costs for gastric cancer [33], S-1 regimens were also less expensive and more convenient than injectable regimens. Furthermore, in our other study of colorectal cancer in an outpatient setting [19], S-1-based regimens were cost saving as compared with 5-FU-based regimens. This result was derived from the analysis including time costs of administration of anticancer drugs. Therefore, our findings suggest that further cost-effectiveness analyses comparing the IRIS regimen with other regimens, taking into account societal costs, time-related costs, and quality of life, are warranted. We hope that our results will help decision makers obtain a more comprehensive view of costs and thereby narrow the gap between clinical trials and actual clinical practice and will help clinicians select regimens for individual patients.

\section{Conclusions}

The cost of the entire course of chemotherapy for advanced colorectal cancer was considerably expensive in Japan. The costs of irinotecan-based regimens such as IRIS and FOLFIRI were lower than those of oxaliplatinbased regimens, such as FOLFOX, and regimens including molecular targeted agents, such as FOLFOX + Bev and FOLFIRI + Bev or Cet. Using lower cost regimens such as IRIS as first-line chemotherapy can lead to a reduction in overall costs for the entire course of chemotherapy, because the duration of first-line treatment was longer than the duration of second and subsequent lines of chemotherapy. The main cost drivers of treatment for advanced colorectal cancer were anticancer drug costs and hospital expenses.

\section{Additional file}

Additional file 1: Schedule of each regimen. (DOCX $15 \mathrm{~kb}$ )

\section{Abbreviations}

FOLFOX: oxaliplatin plus 5-fluorouracil and leucovorin; FOLFIRI: irinotecan plus 5-fluorouracil and leucovorin; XELOX: oxaliplatin plus capecitabine; IRIS: irinotecan plus S-1; IFL: irinotecan plus 5-fluorouracil and leucovorin; 5-FU: 5-fluorouracil; LV: leucovorin; S-1: tegafur, gimeracil, and oteracil combination; UFT: tegafur and uracil combination; Bev: bevacizumab; Cet: cetuximab.

\section{Competing interests}

Shuichi Yajima is an employee of Taiho Pharmaceutical Co., Ltd. The other authors have no competing interests to declare. This research was not funded by any company.

\section{Authors' contributions}

SY, H Shimizu, and H Sakamaki contributed to the study conception and design. SI, NI and JM participated in the study design. SY and H Shimizu were involved in data acquisition. SY, H Shimizu, H Sakamaki and NI were involved in data analysis and interpretation. SY drafted the manuscript and revised it critically for important intellectual content. H Shimizu, H Sakamaki, $\mathrm{SI}, \mathrm{NI}$ and JM helped to draft the manuscript and revise it. All authors read and approved the final manuscript.

\section{Acknowledgments}

The authors would like to express their appreciation to the members of the patient billing office of Showa University Hospital who provided the billing data on the patients for this study.

\section{Author details}

${ }^{1}$ Department of Health Policy and Management, School of Medicine, Keio University, 35, Shinanomachi, Shinjuku-ku, Tokyo 160-8582, Japan. ${ }^{2}$ Taiho Pharmaceutical Co., Ltd., 1-27, Kandanishiki-cho, Chiyoda-ku, Tokyo 101-8444, Japan. ${ }^{3}$ Department of Pharmacy Services, Showa University Hospital, 1-5-8, Hatanodai, Shinagawa-ku, Tokyo 142-8666, Japan. ${ }^{4}$ School of Management, Tokyo University of Science, 500, Shimokiyoku, Kuki, Saitama 346-8512, Japan. ${ }^{5}$ Department of Pharmaceutical Sciences, School of Pharmacy, International University of Health and Welfare, 2600-1 Kitakanemaru, Otawara, Tochigi 324-8501, Japan. ${ }^{6}$ Keio University, 5-29-20-409 Shiba, Minato-ku, Tokyo 108-0014, Japan. ${ }^{7}$ Department of Hospital Pharmaceutics, School of Pharmacy, Showa University, 1-5-8, Hatanodai, Shinagawa-ku, Tokyo 142-8555, Japan. 
Received: 9 February 2015 Accepted: 24 December 2015 Published online: 04 January 2016

\section{References}

1. International Agency for Research on Cancer: GLOBOCAN 2012 Estimated cancer incidence, mortality and prevalence worldwide in 2012. 2012. http://globocan.iarc.fr/Pages/fact_sheets_population.aspx. Accessed 15 Jan 2015.

2. National Cancer Center, information cancer: Monitoring of Cancer Incidence in Japan. 2010. http://ganjoho.jp/en/professional/statistics/table_download. html. Accessed 15 Jan 2015.

3. Ministry of Health, Labour and Welfare, Statistics and Information Department: Vital statistics in 2013 (in Japanese). 2013. http://www.mhlw.go. j.j/toukei/saikin/hw/jinkou/kakutei13/dl/00 all.pdf. Accessed 15 Jan 2015.

4. Tournigand C, Andre T, Achille E, Lledo G, Flesh M, Mery-Mignard D, et al. FOLFIRI followed by FOLFOX6 or the reverse sequence in advanced colorectal cancer: A randomised GERCOR study. J Clin Oncol. 2004;22(2):229-37.

5. Saltz LB, Clarke S, Díaz-Rubio E, Scheithauer W, Figer A, Wong R, et al. Bevacizumab in combination with oxaliplatin-based chemotherapy as firstline therapy in metastatic colorectal cancer: a randomized phase III study. J Clin Oncol. 2008;26(12):2013-9.

6. National Comprehensive Cancer Network (NCCN): Clinical practice guidelines in oncology - Colon cancer - version 2.2015. 2015. http://www. nccn.org/professionals/physician_gls/PDF/colon.pdf. Accessed 15 Jan 2015

7. National Institute for Health and Care Excellence (NICE): clinical guideline 131 - the diagnosis and management of colorectal cancer. 2014. http:// www.nice.org.uk/guidance/cg131/. Accessed 15 Jan 2015.

8. Japanese Society for Cancer of the Colon and Rectum: JSCCR guidelines 2010 for the treatment of colorectal cancer (in Japanese). 2010. http://www. jsccr.jp/guideline/2010/index_guide.html. Accessed 15 Jan 2015.

9. Cassidy J, Clarke S, Díaz-Rubio E, Scheithauer W, Figer A, Wong R, et al. Randomized phase III study of capecitabine plus oxaliplatin compared with fluoro- uracil/folinic acid plus oxaliplatin as first-line therapy for meta- static colorectal cancer. J Clin Oncol. 2008;26(12):2006-12.

10. Rothenberg ML, Cox JV, Butts C, Navarro M, Bang YJ, Goel R, et al. Capecitabine plus oxaliplatin (XELOX) versus 5 -fluorouracil/folinic acid plus oxaliplatin (FOLFOX-4) as second-line therapy in metastatic colorectal cancer: a randomized phase III noninferiority study. Ann Oncol. 2008; 19(10):1720-6.

11. Goto A, Yamada Y, Yasui H, Kato K, Hamaguchi T, Muro K, et al. Phase II study of combination therapy with S-1 and irinotecan in patients with advanced colorectal cancer. Ann Oncol. 2006;17(6):968-73.

12. Muro K, Boku N, Shimada Y, Tsuji A, Sameshima S, Baba H, et al. Irinotecan plus S-1 (IRIS) versus fluorouracil and folinic acid plus irinotecan (FOLFIRI) as second-line chemotherapy for metastatic colorectal cancer: a randomised phase 2/3 non-inferiority study (FIRIS study). Lancet Oncol. 2010;11:853-60.

13. Ministry of Health, Labour and Welfare: Estimates of national medical care expenditure in 2012 (in Japanese). 2012. http://www.mhlw.go.jp/toukei/ saikin/hw/k-iryohi/12/dl/kekka.pdf. Accessed 15 Jan 2015.

14. Miyazaki Y, Harada T, Akase T. Cost-minimization analysis of sequence changes between FOLFIRI and FOLFOX6 therapy for advanced colorectal cancer in japan. Clin Ther. 2009;31(Theme Issue):2433-41.

15. Shiroiwa T, Fukuda T, Tsutani K. Cost-effectiveness analysis of bevacizumab combined with chemotherapy for the treatment of metastatic colorectal cancer in japan. Clin Ther. 2007;29(10):2256-67.

16. Ajima H, Ogata H, Fujita K. Clinical and economic evaluation of first-line therapy with FOLFIRI or modified FOLFOX6 for metastatic colorectal cancer. Jpn J Clin Oncol. 2010;40(7):634-8.

17. Garrison Jr LP, Neumann PJ, Erickson P, Marshall D, Mullins CD. Using realworld data for coverage and payment decisions: the ISPOR real-world data task force report. Value Health. 2007:10(5):326-35.

18. Berger ML, Martin BC, Husereau D, Worley K, Allen JD, Yang W, et al. A questionnaire to assess the relevance and credibility of observational studies to inform health care decision making: an ISPOR-AMCP-NPC good practice task force report. Value Health. 2014;17:143-56.

19. Shimizu H, Ikeda S, Sakamaki H, Yajima S, Ikegami N, Murayama J. Costminimization analysis of S-1 plus irinotecan (IRIS) versus oxaliplatin or irinotecan plus fluorouracil and folinic acid (mFOLFOX6 or FOLFIRI) in patients with advanced colorectal cancer. Japanese Journal of Pharmaceutical Health Care and Sciences. 2015;41(3):153-62.
20. Ikegami N, Anderson GF. In Japan, all-payer rate setting under tight government control has proved to be an effective approach to containing costs. Health Aff (Millwood). 2012;31(5):1049-56.

21. Ministry of Health, Labour and Welfare, Ministry of Education, Culture, Sports, Science and Technology: Ethical Guidelines for Epidemiological Studies (in Japanese). 2002 (Amended 2008). http://www.mhlw.go.jp/ general/seido/kousei/i-kenkyu/ekigaku/0504sisin.html. Accessed 15 Jan 2015.

22. Ministry of Health, Labour and Welfare: Summary report of basic survey on wage structure (nationwide) 2012. 2012. http://www.mhlw.go.jp/english/ database/db-l/dl/24gaikyo_zenkoku_Eng.pdf. Accessed 15 Jan 2015.

23. Mullins CD, Hsiao FY, Onukwugha E, Pandya NB, Hanna N. Comparative and cost-effectiveness of oxaliplatin-based or irinotecan-based regimens compared with 5-fluorouracil/ leucovorin alone among US elderly stage IV colon cancer patients. Cancer. 2012;15:3173-81.

24. Tumeh JW, Shenoy PJ, Moore SG, Kauh J, Flowers C. A markov model assessing the effectiveness and cost-effectiveness of FOLFOX compared with FOLFIRI for the initial treatment of metastatic colorectal cancer. Am J Clin Oncol. 2009;32(1):49-55.

25. Lloyd Jones M, Hummel S, Bansback N, Orr B, Seymour M. A rapid and systematic review of the evidence for the clinical effectiveness and costeffectiveness of irinotecan, oxaliplatin and raltitrexed for the treatment of advanced colorectal cancer. Health Technol Assess. 2001;5(25):1-128.

26. Hind D, Tappenden P, Tumur I, Eggington S, Sutcliffe P, Ryan A. The use of irinotecan, oxaliplatin and raltitrexed for the treatment of advanced colorectal cancer: systematic review and economic evaluation. Health Technol Assess. 2008:12(15):iii-ix. xi-162

27. Tappenden P, Jones R, Paisley S, Carroll C. The cost-effectiveness of bevacizumab in the first-line treatment of metastatic colorectal cancer in England and Wales. Eur J Cancer. 2007;43:2487-94.

28. Lee E, Revil C, Ngoh CA, Lister J, Kwon J. Clinical and cost effectiveness of bevacizumab + FOLFIRI combination versus FOLFIRI alone as first-line treatment of metastatic colorectal cancer in South Korea. Clinical Therapeutics. 2012;34(6):1408-19.

29. National Institute for Health and Care Excellence (NICE): technology appraisal guidance118. Bevacizumab and cetuximab for the treatment of metastatic colorectal cancer. 2007. http://www.nice.org.uk/guidance/ta118/. Accessed 15 Jan 2015.

30. Chu E, Schulman KL, McKenna Jr EF, Cartwright T. Patients with locally advanced and metastatic colorectal cancer treated with capecitabine versus 5-fluorouracil as monotherapy or combination therapy with oxaliplatin: a cost comparison. Clin Colorectal Cancer. 2010;9(4):229-37.

31. Ward SE, Kaltenthaler E, Cowan J, Marples M, Orr B, Seymour MT. The clinical and economic benefits of capecitabine and tegafur with uracil in metastatic colorectal cancer. Br J Cancer. 2006;95:27-34.

32. Perrocheau G, Bennouna J, Ducreux M, Hebbar M, Ychou M, Lledo G, et al. Cost-minimisation analysis in first-line treatment of metastatic colorectal cancer in France: XELOX versus FOLFOX-6. Oncology. 2010;79:174-80.

33. Sakamaki H, Ikeda S, Yajima S, Ikegami N, Tanaka K, Shimizu H, et al. Cost-utility analysis of the oral fluoropyrimidine $\mathrm{S}-1$ versus conventional intravenous chemotherapy in advanced or recurrent gastric cancer. The Open Health Services and Policy Journal. 2009;2:26-33.

\section{Submit your next manuscript to BioMed Central and we will help you at every step:}

- We accept pre-submission inquiries

- Our selector tool helps you to find the most relevant journal

- We provide round the clock customer support

- Convenient online submission

- Thorough peer review

- Inclusion in PubMed and all major indexing services

- Maximum visibility for your research

Submit your manuscript at www.biomedcentral.com/submit 